\title{
How Semantics is Embodied through Visual Representation: Image Schemas in the Art of Chinese Calligraphy
}

\author{
TIFFANY YING-YU LIN \\ National Taiwan University \\ I-HSUAN CHEN \\ University of California, Berkeley
}

\section{Introduction}

This study aims to investigate abstract reasoning and embodied cognition through the analysis of image schemas and conceptual metaphors in the interplay of art and language.

Chinese calligraphy is noteworthy due to its unique embodied characteristics and image-schematic representations of visual art and language. The art of Chinese calligraphy not only represents the visual forms of Chinese characters but also conveys meanings, emotion, and style, demonstrating the aesthetics of language and art.

By analyzing image schemas and metaphors in classical works of art, this paper shows how semantics is conceptualized and embodied through visual representation of Chinese calligraphy. In this study, we examine how semantics is visualized within the topological structure of cognitive mechanisms of a CONTAINER schema, the crucial image schema that structures the conceptualization of spatial relation concepts. This paper proposes that the CONTAINER schema, the BALANCE schema, the FORCE schema, as well as the metaphors SIGNIFICANCE IS SIZE and MIND IS A BODY, which may motivate the calligrapher's creative process, underlie the art of Chinese calligraphy.

\footnotetext{
* The previous version of this article was presented at the $38^{\text {th }}$ annual meeting of Berkeley Linguistics Society, in Berkeley, U.S.A., 2012. We are grateful to the participants there, especially our chair, Prof. Eve Sweetser, for their questions and valuable comments. Sincere thanks are also due to, Liangyong Peng, the researcher in Chinese art history, for the fruitful discussions we had at National Taiwan University. We are indebted to the editor for offering insightful comments and suggestions. Any errors remaining herein are solely our responsibility.
} 
Our findings show that the semantics in Chinese calligraphy is construed through the visual forms of Chinese characters, whereas the layout and spatial distribution between the characters express the pragmatics, including emphatic functions, rhetorical readings, and informative purposes. How viewers interpret the art of Chinese calligraphy is based on the most salient images, such as sizes of characters, degrees of boldness, and spatial balance. Viewers use the visual cues as the cognitive anchors to get their interpretation based on primary metaphors and schemas. In this study, we provide an analysis to explore how such primary cognitive mechanism links visual perception and semantic/pragmatic recognition.

\section{$1 \quad$ Background}

The main tenet of Conceptual Metaphor Theory (Lakoff and Johnson 1980, 1999) is that conceptual mechanisms arising from our sensorimotor experience and neural structures, including image schemas, metaphors, and other embodied imaginative structures, help us conceptualize and experience our world. The mechanisms of image schemas operating with conceptual metaphors enable human beings to employ the logic of our sensory-motor experience to perform high-level cognitive operations for abstract entities and domains (Johnson 2005: 26). As previous studies on cognitive science show, the process of image-schematic and metaphor-based understanding has been demonstrated for concepts in different fields, including mathematics (Lakoff and Nuñez 2000), law (Winter 2001), morality (Johnson 1993), analogical problem solving (Craig, Nersessian and Catrambone 2002), scientific causality (Lakoff and Johnson 1999), psychology (Fernandez-Duque and Johnson 1999; Gibbs and Colston 1995).

However, few researches focused on abstract reasoning and theorizing in the interface between art and language, therefore, this study aims to bridge the gap by providing an explanation for abstract reasoning and embodied cognition in the field of art and language based on image-schematic and metaphor-based analysis. In the next section, we will introduce the data we focus on in this paper.

\section{$2 \quad$ Data}

The data is selected from two classical pieces of art by calligrapher Master Wang Xi-zhi (303-361 AD, Jin Dynasty) whose works have been studied as examples to learn and practice the art of calligraphy.

The two representative styles of Master Wang are the frame script, Lantingjixu (lit., 'Preface to the Poems Composed at the Orchid Pavillon'), and the cursive script, Shiqitie (lit., 'The Seventeenth Script'), as shown in Figure 1. 


\section{Embodiment and Image Schema in Chinese Calligraphy}

Frame script is characterized for its neatness, equal balance, and clearness of each stroke. In contrast, cursive script is famous for its running stroke and simplified structure of characters. A comparison of the two distinct styles in terms of visual cues can help us better capture what roles primary schemas and metaphors play.

Figure 1: Excerpts of the two representative styles of Master Wang

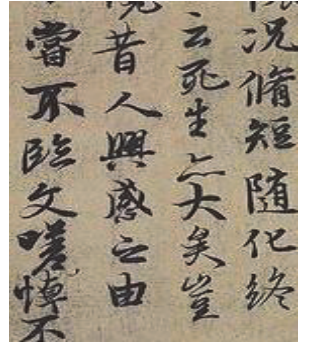

The Frame script: Lantingjixu

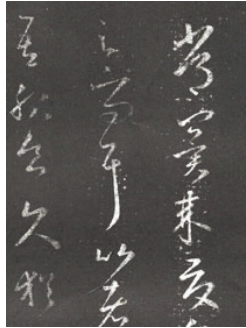

The Cursive script: Shiqitie

Lantingjixu is the introduction to a collection of poems written by several poets in a summer gathering at a place called Lanting. Master Wang described the time, location, and purpose of the Spring festival, revealing his viewpoints about life, and lamenting the fast passing of time.

As for Shiqitie, it is a letter correspondence with a friend written by Master Wang. He recoded the current status of himself and his family, and included his own thoughts on life in this correspondence.

In the next section, we will discuss how semantics and pragmatics are embodied through visual representation in the two classical pieces of art in Chinese calligraphy.

\section{Connection between image schemas, metaphors, and Chinese calligraphy}

In this study, we suggest that the visual form and meanings of Chinese calligraphy are conceptualized and visualized through the CONTAINER schema, having the gestalt structure of an interior, an exterior, and a boundary. The visual form that consists of lines and brush strokes is construed as the boundary, the semantics is construed as the interior, and the space allocation is construed by the exterior of visual forms. Specifically, the semantics is construed within the interior, while the pragmatic function, such as emphatic functions, rhetorical readings, and informative purposes, are reached by the spatial allocation of the exterior of each visual form. In the following analysis, we will examine the schematic characteristics with examples. 
Tiffany Ying-yu Lin and I-Hsuan Chen

\subsection{The link between the CONTAINER schema and semantics}

In Chinese calligraphy, different sizes of visual forms reveal topological characteristic of the CONTAINER schema. Our analysis shows that when the interior of the CONTAINER schema is significant, the boundary is enlarged. In other words, the size of a visual form can be larger when the meaning or pragmatic function of the interior is significant, for example, when there is a key word with core semantics in the paragraph or a pragmatic marker with important discourse function in the context. As shown in Figure 2, the characters marked in the red square are those with significant meanings, important grammatical functions, or emphasized contents. For example, the character at the left-top corner, ye, the grammatical word with pragmatic functions, is enlarged to emphasize the end of a statement with a pause of the emotion.

Figure 2: Excerpt of cursive script from Shiqitie
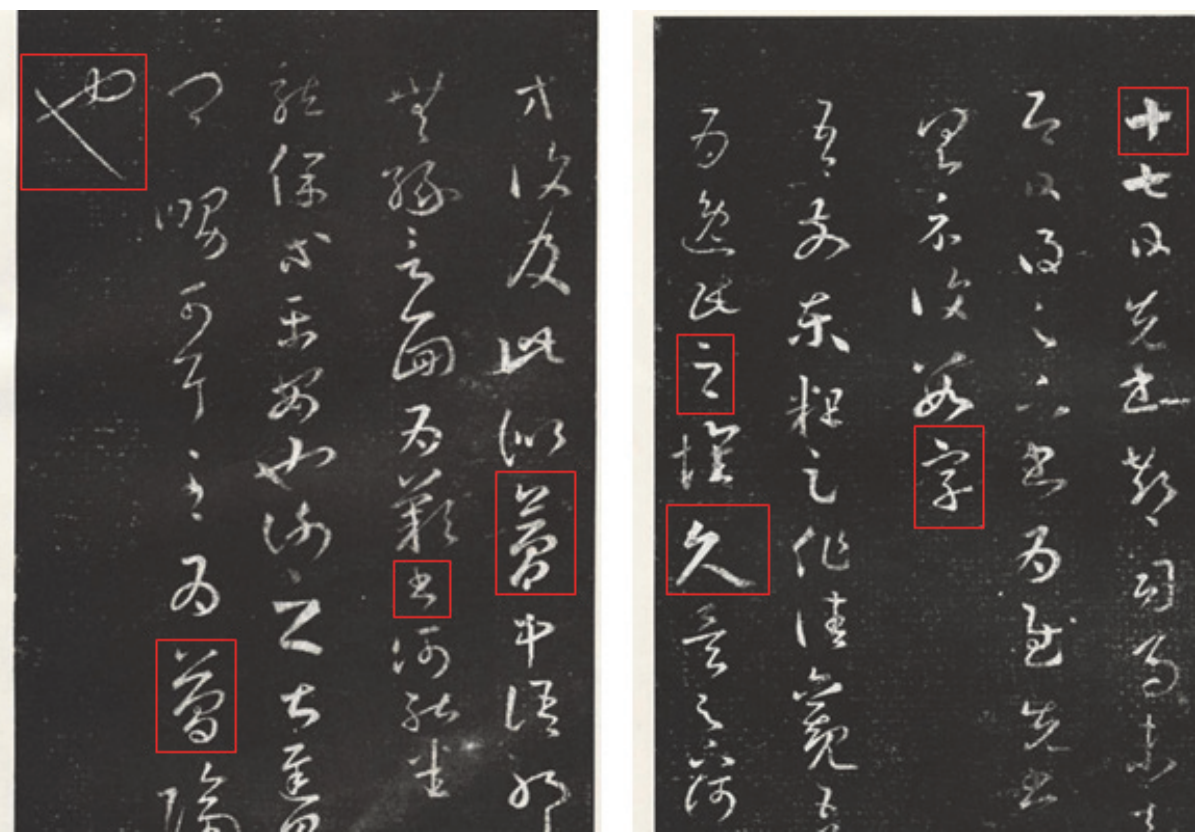

We claim that the primary metaphor, SIGNIFICANCE IS SIZE, motivates the visualization of topological characteristic in Chinese calligraphy, which strengthens the natural flow in cursive script. However, some free variations of size are found in cursive script as well. 


\section{Embodiment and Image Schema in Chinese Calligraphy}

\subsection{Visual cues construed by the BALANCE schema}

As previously mentioned, the space allocation of visual forms is construed by the exterior of CONTAINER schema in Chinese calligraphy. The space allocated in the exterior creates the scale and the atmosphere in the art work. We propose the BALANCE schema that involves "a symmetrical or proportional arrangement of forces around a point or axis (Johnson 1987: 85-98)" is incorporated in the exterior to keep the visual cues, including 'visual weight' and 'visual force,' in balance while sustaining the natural flow in the art of Chinese calligraphy.

Figure 3: Excerpt of cursive script from Shiqitie
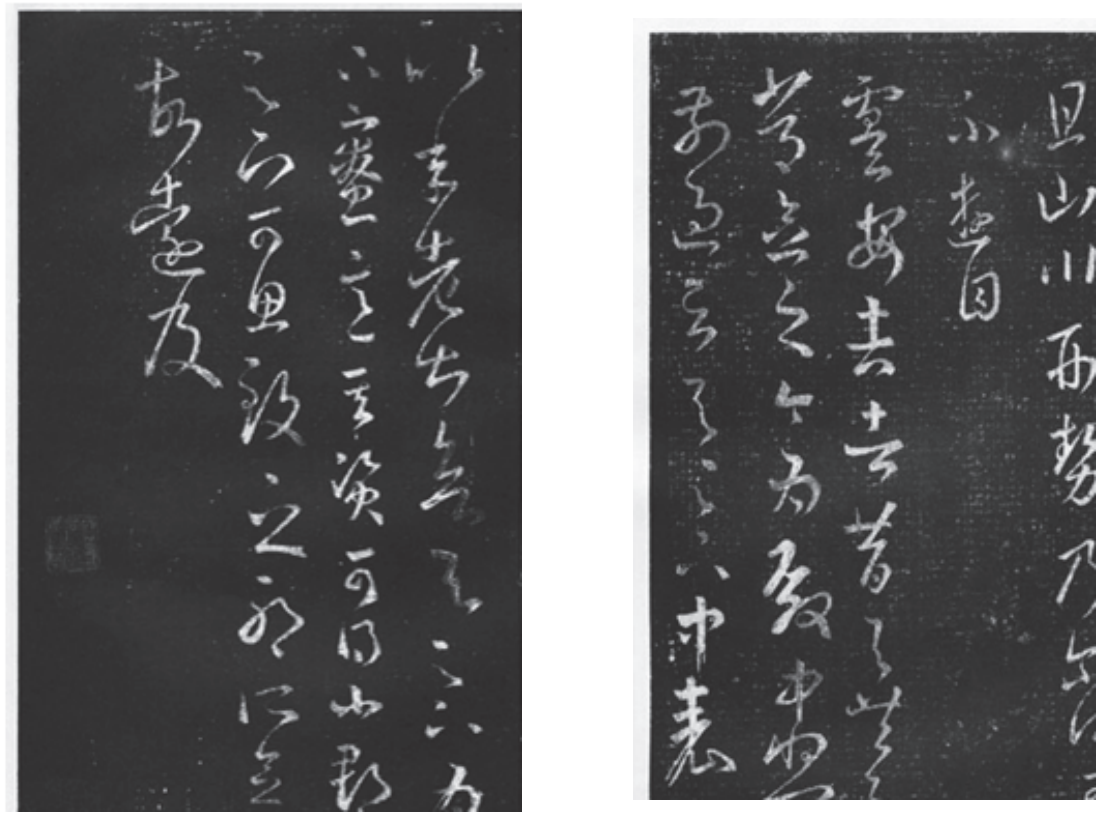

As Figure 3 shows, the curving lines in the exterior, a natural segment of emotion of the calligrapher, not only connect the visual forms to maintain the coherence and balance but also reveal the speed and flow of the visual force. The curving lines and forms in the cursive script are connected depending on the coherence and rhythm in the context. The stylistic forms and natural flow of the atmosphere visually represent the indivisible and context-dependent features of cursive script. 
Tiffany Ying-yu Lin and I-Hsuan Chen

\subsection{The FORCE schema and embodiment of emotion}

While examining bold visual forms in Chinese calligraphy in Section 3.1 and 3.2, we also claim that FORCE schema is involved in the process of creation. They motivate different degrees of boldness in the lines and strokes. The writing process of Chinese calligraphy requires great control of the force from the wrist, arm, and body. As a result, the boldness of lines and strokes can be viewed as the representation of 'visual force' in the BALANCE schema, which indicates strong intensity of force as the calligrapher is writing. Our data shows that the bolded visual forms are mostly 'emotion words' that express the calligrapher's personal feelings and attitudes, for example, the bolded words for 'painful' and 'sigh with grief,' as indicated by the arrows in Figure 4.

Figure 4: Excerpt of frame script from Lantingjixu

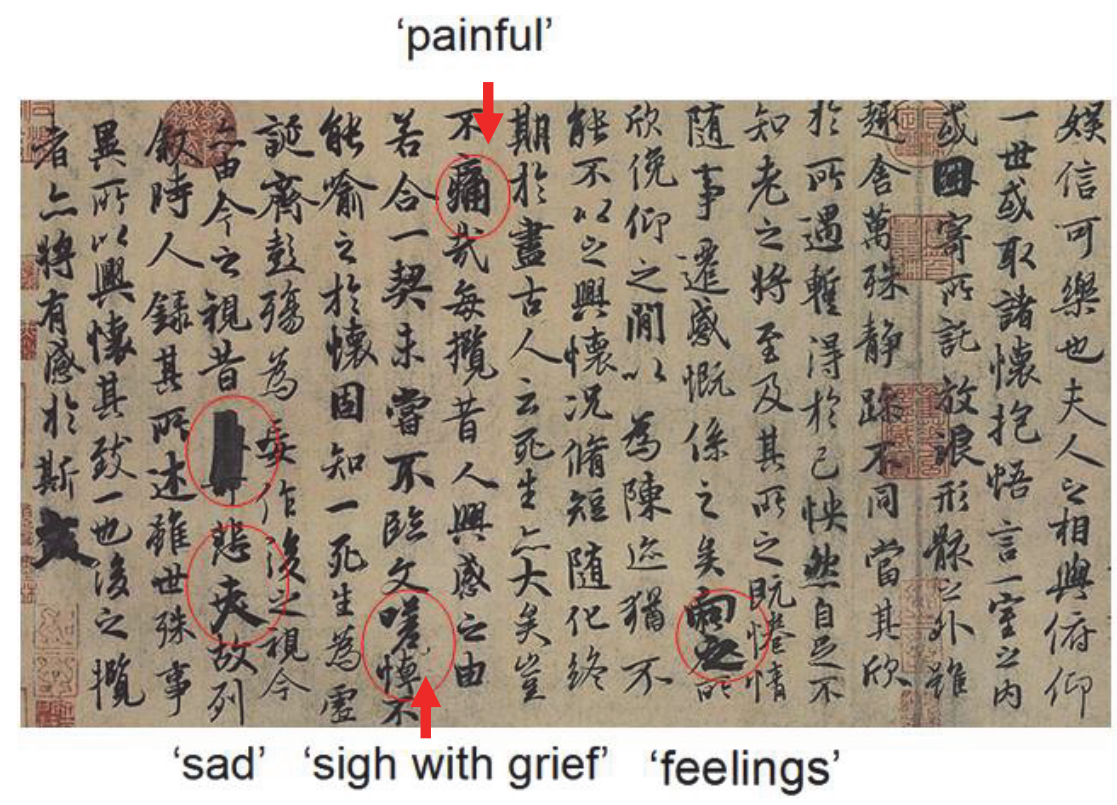

The erasing trace and revising marks left with the emotion words, 'painful', 'sad', 'sigh with grief', 'feelings' shown in the circles in Figure 4 is another crucial feature that may reflect calligrapher's emotion during the creation process. As Figure 5 shows, the content of the right part (divided by the line in Figure 5) focused more on objective description, such as, time, location, and participants in the festival, while the left part focused more on Master Wang's comments and personal feelings. More erasing traces and revising marks are found in the left part where calligrapher Master Wang was expressing his feelings and philosophy of life. In comparison, very few erasing traces and revising marks are found in the right part where the content mainly describes the event. 


\section{Embodiment and Image Schema in Chinese Calligraphy}

Figure 5: The whole script of Lantingjixu

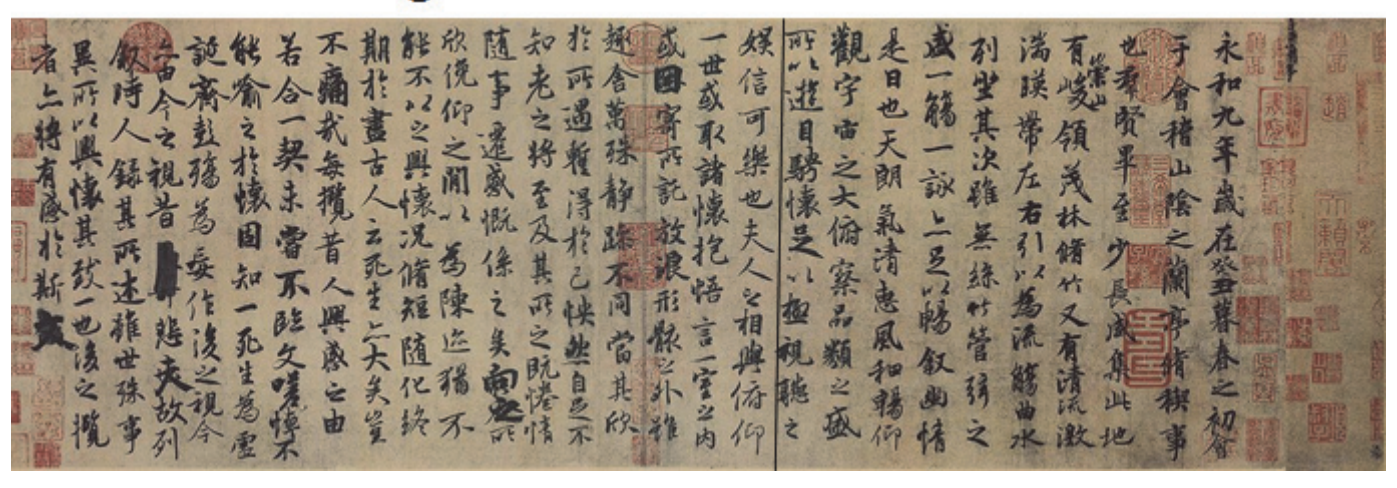

Subjective emotion/ comment

Objective description

Our analysis shows that the emotion and feelings of Master Wang have been visualized through boldness, erasing trace, and revising marks in his art works. As the layout of characters reflects the force from a physical body movement, our finding suggests that the metaphor, MIND IS A BODY, motivate such representation of 'visual force,' which demonstrates vividly how mind and emotion can be conceptualized as body movements of the creating process in Chinese calligraphy.

In this section, our analysis highlights the correlation between visual representation and semantics in Chinese calligraphy. We propose that the complex conceptual structure of the CONTAINER SCHEMA, the BALANCE SCHEMA, the FORCE SCHEMA, and metaphors, SIGNIFICANCE IS SIZE, MIND IS A BODY, underlie the art of Chinese calligraphy.

\section{Discussion and Conclusion}

This study shows how the conceptualization of a calligrapher's emotion and feelings are visualized through different schemas and metaphors in the embodied art work. The embodied experience of the Chinese calligrapher represented visually in art has provided us new insights on abstract thinking and reasoning in the interface between art and language. This study concludes that the topological conceptual structure of the CONTAINER schema, the BALANCE schema, the FORCE schema, and the metaphors, SIGNIFICANCE IS SIZE, MIND IS A BODY, which underlie the art of Chinese calligraphy, may motivate the visual representations and creating process. We propose that the embodiment of abstract concepts and meanings through visualization can be demonstrated in the art of Chinese calligraphy. The focus of this paper is on the connection between visual representation and semantics in Chinese calligraphy. What bridges the two is primary cognition mechanism including image schemas and primary metaphors. Chinese characters can be conceptualized as the CONTAINER schemas, which 


\section{Tiffany Ying-yu Lin and I-Hsuan Chen}

function as a salient perceptual cue for viewers to get the emphasis of the contents. The spatial allocation also plays a role to provide pragmatic inferences for viewers. These primary metaphors and schemas can be viewed as cognitive anchors for viewers to transfer their visual perception to meaning interpretation. Besides the visual effects based on Chinese characters, the representation of Chinese calligraphy also embodies and captures calligraphers' subjective viewpoints and emotions via the FORCE schema. Our analysis indicates that the interpretation of Chinese calligraphy involves a set of primary cognition mechanism from both viewers and artists.

From the perspective of a cognitive linguistic analysis, this paper highlights the correlation between visual perception and semantics, hoping to contribute to a body-based theory of conceptualization and reasoning in the field of language, art, and cognition. Through this study, we hope to shed light on the cross-modal features of image schema and provide a better understanding of abstract inferential structure in different modes.

\section{References}

Craig, D., N. Nersessian, and R. Catrambone. 2002. Perceptual Simulation in Analogical Problem Solving. In L. Magnani and N. Nersessian, eds., Model-Based Reasoning: Science, Tecnology, Values, 167-190. New York. Kluwer.

Fernandez-Duque, Diego, and Mark Johnson. 1999. Attention Metaphors: How Metaphors Guide the Cognitive Psychology of Attention. Cognitive Science 23: 83-116.

Gibbs, Raymond W., Jr., and Herbert Colston. 1995. The Psychological Reality of Image Schemas and their Transformations. Cognitive Linguistics 6: 347-378.

Johnson, Mark. 1987. The Body in the Mind: The Bodily Basis of Meaning, Imagination, and Reason. Chicago: University of Chicago Press.

Johnson, Mark. 1993. Moral Imagination: Implications of Cognitive Science for Ethics. Chicago: University of Chicago Press.

Johnson, Mark. 2005. The Philosophical Significance of Image Schemas. In B. Hampe and J. E. Grady, eds., From Perception to Meaning. Image Schemas in Cognitive Linguistics, 15-33. Berlin/New York: Mouton de Gruyter.

Lakoff, George, and Mark Johnson. 1980. Metaphors We Live By. Chicago: University of Chicago. 


\section{Embodiment and Image Schema in Chinese Calligraphy}

Lakoff, George, and Mark Johnson. 1999. Philosophy in the Flesh: The Embodied Mind and its Challenge to Western Thought. New York: Basic Books.

Lakoff, George, and Rafael Nuñez. 2000. Where Mathematics Comes From: How the Embodied Mind Brings Mathematics into Being. New York: Basic Books.

Winter, Steven. 2001. A Clearing in the Forest: Law, Life, and Mind. Chicago: University of Chicago Press.

Tiffany Ying-Yu Lin

3F, Le-xue Building, No.1, Sec. 4, Roosevelt Road, Taipei, Taiwan d98142002@ntu.edu.tw

\section{I-Hsuan Chen}

1203 Dwinelle Hall, UC Berkeley, Berkeley, CA 94720

ihsuanchen@berkeley.edu 